\title{
ÉTUDE DE LA LIBÉRATION DE L'AZOTE, DU PHOSPHORE ET DE L'ACIDE SIALIQUE NON PROTÉIQUES, AU COURS DE L'ACTION DE LA PRÉSURE SUR LA CASÉINE (1)
}

\author{
CII. ALAIS ( $\left.{ }^{2}\right)$ \\ Centre national de Recherches zootechniques, \\ Institut national de la Recherche agronomique, Jouy-en-Josas (Seine-et-Oise)
}

SOMMAIRE

La libération de substances azotées non protéiques, au cours de l'action de la présure sur la caséine, a été étudiée. Ces substances sont séparées du mélange réactionnel au moyen de l'acide trichloracétique.

La libération de l'azote non protéique, qui se traduit par une courbe NPN $f(t)$ caractéristique, est liée au raccourcissement du temps de coagulation du lait : ce temps devient nul lorsque la réaction enzymatique est achevée.

La quantité d'azote non protéique n'est proportionnelle à la concentration du substrat que pour les faibles valeurs. L'adsorption des substances solubles sur le précipité explique les anomalies constatées.

Les résultats comparatifs obtenus avec la caséine $x$ et la caséine entière dont elle provient, permettent de calculer une proportion de I9 p. Ioo de la caséine $\%$ dans la caséine entière.

La présure libère le phosphore et l'acide sialique, solubles dans l'acide trichloracétique à I 2 p. ıoo, à la même vitesse que l'azote.

On a conclu que la réaction étudiée, avec libération du caséino-glycopeptide, est bien la réaction primaire spécifique de la présure, indispensable à la coagulation.

\section{INTRODUCTION}

La coagulation du lait par la présure est consécutive à une protéolyse limitée de la caséine, au cours de laquelle se forment une partie insoluble en présence d'ions $\mathrm{Ca}^{++}$, la "paracaséine », et une partie soluble, en faible proportion, la " protéose de

(1) Cette étude fait partie des recherches présentées dans une thèse soutenue en i 962 devant la Faculté des Sciences de Paris.

$\left({ }^{2}\right)$ Adresse actuelle : Institut de Biochimie, Faculté des Sciences, Orsay (Seine-et-Oise). 
Hammarstex " (I874). BerRidge (I942) a distingué deux stades successifs dans ce phénomène :

a) Un stade enzymatique au cours duquel se produit l'attaque de la caséine parla présure. Cette réaction n'exige pas la présence d'ions $\mathrm{Ca}^{++}$et possède un faible coefficient de température; elle se produit encore à une vitesse notable à $\mathrm{O}^{\circ} \mathrm{C}$; on l'appelle également " réaction primaire".

b) Un stade de coagulation proprement dite qui concerne la plus grande partie des substances formées au cours du premier stade. Cette deuxième réaction, ou "réaction secondaire ", possède un coefficient de température élevé ( I,5 par degrẻ C), comme une réaction de dénaturation; elle est extrêmement lente au-dessous de Io ${ }^{\circ} \mathrm{C}$.

Nous avons précédemment montré (ALAIs et al., I953) que l'on peut dissocier la réaction primaire de la réaction secondaire en faisant agir la présure sur des solutions sodiques de caséine et que l'on peut suivre le cours de la protéolyse en mesurant la libération de l'azote non protéique, soluble dans l'acide trichloracétique, en fonction du temps. Nous avons également montré (ALAIs, I956) que le filtrat trichloracétique à I2 p. Ioo de concentration finale contient, presqu'à l'état de pureté, une substance glycopeptidique que nous arons appelée "NPN-I2 p. I00 ", puis "caséinoglycopeptide ". WAIGH et $v$. HIPPEL (I956) ont émis 1'hypothèse que la présure réagit spécifiquement sur la caséine $x$.

Nous arons poursuivi l'étude de la libération des substances non protéiques afin de préciser leur relation avec la réaction primaire et de parvenir à une meilleure connaissance de cette réaction qui précède la coagulation.

\section{MATÉRIEL F'T MÉTHODES}

\section{Sulsirat}

Nous avons utilisé comparativement la caséine entière, la caséine $\alpha$ et la caséine $x$ ae vache. Quelques expériences ont été faites avec le lait de vache afin de mesurer le temps de coagulation parallèlement au dosage de l'azote non protéique (NPN).

Lait : lait de vache frais et écrémé par centrifugation.

Caséine entière : obtenue par précipitations répétées du lait à pII 4,6 .

Caséine $\alpha$ : obtentue par fractionnement de la caséine entière au moyen de l'urée, selon HIPP et coll. (1952)

Caséine $x$ : obtenue en suivant la méthode de MCKenZIE et WaKE (I96I).

Pour être soumises à l'action de la présure, les caséines sont dissoutes dans l'eau à pII 6,8, avec addition de $\mathrm{NaOH} 0, \mathrm{I} \mathrm{N}$.

\section{Ensyme}

Présure cristallisée extraite des caillettes de veaux (ALAIS, 1955). La réaction de la présure sur les solutions de caséine a lieu à $25^{\circ} \mathrm{C}$ et sur le lait, à $2^{\circ} \mathrm{C}$.

\section{Méthodes analytiques}

L'azote est dosé par micro-Kjeldahl ; le phosphore par la méthode de Bamman et coll. (1948) après digestion par l'acide sulfurique concentré ; l'acide sialique par la méthode à l'acide thiobarbiturique de WARREN (1959), après hydrolyse par l'acide sulfurique o, I N (SvENNERHOLM, I958) et en 
prenant comme standard l'acide- $\mathrm{N}$ acétylneuraminique cristallisé extrait de la caséine de vache (Ribadeau Dumas et Alais, r96r). La méthode de Warren est applicable en présence d'acide trichloracétique; des essais préliminaires nous ont montré que l'acide sialique n'est pas détruit.

Les courbes NPN $f(t)$ sont établies en dosant l'azote du filtrat trichloracétique à différents temps d'action de la présure sur les solutions de caséine ou sur le lait. Les courbes de phosphore et d'acide sialique non protéiques sont établies de la même manière. Les conditions de la déprotéinisation par l'acide trichloracétique (TCA) sont précisées à chaque expérience.

\section{RÉSULTATS}

\section{I.- Relation entre la libération de l'azote non protéique (NPN) et le temps de coagulation du lait}

Ia figure I représente la courbe de libération de l'azote non protéique dans du lait à $\mathrm{pH} 6,7$ sous 1'action de la présure. Le mélange réactionnel est maintenu à $2^{\circ} \mathrm{C}$ afin d'empêcher la coagulation du lait. Les traits horizontaux représentent le temps

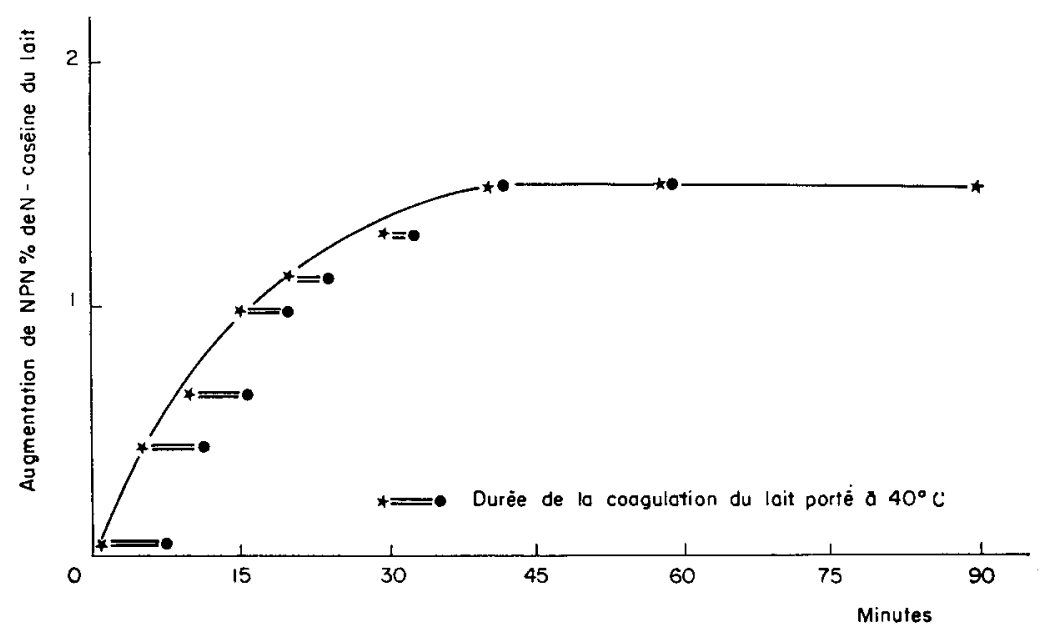

FIG. I. - Libération de l'azote non protéique (NPN) dans le lait et raccourcissement du temps de coagulation par la présure $e: 0,04 \mathrm{UP} / \mathrm{ml}\left(0,5 \mu \mathrm{g}\right.$ de présure cristallisée) réaction à $2^{\circ} \mathrm{C}$. TCA : I $2 \%$

de coagulation d'un petit volume de mélange brusquement porté à $40^{\circ} \mathrm{C}$ au moment de chaque prélèvement pour le dosage de l'azote non protéique. Ce temps de coagulation va en décroissant et devient pratiquement nul au début du palier formant la deuxième partie de la courbe.

Il existe donc une relation entre le raccourcissement du temps de coagulation et la libération de l'azote non protéique soluble dans I2 p. xoo de TCA. 
II. - Infuence de la concentration du substrat sur la quantité d'azote non protéique

Le tableau I résume les résultats d'expériences faites avec 4 solutions de caséine, dont les concentrations sont comme I - 3,2 - Io - I2, et avec quatre concentrations de TCA, la quantité de présure étant constante $(0,3 \mathrm{UP} / \mathrm{ml}$, soit $4 \mu \mathrm{g} / \mathrm{ml}$ ). Les dosages sont faits après $25 \mathrm{mn}$ d'action de présure (début du palier de la courbe à I2 p. IOo de TCA).

TABLEAU I

Influence de la concentration des solutions de caséine et de la concentration de T'CA sur la quantité de $N P N$. (Augmentation du NPN après action de la présure, en $\mu \mathrm{g} / \mathrm{ml}$ de solution de caséine, et en p. Ioo de $\mathrm{N}$-caséine)

\begin{tabular}{|c|c|c|c|c|c|c|c|c|}
\hline \multirow{3}{*}{$\begin{array}{c}\text { Concentration finale } \\
\text { en TCA }\end{array}$} & \multicolumn{8}{|c|}{ Solution de caséine à } \\
\hline & \multicolumn{2}{|c|}{$0,45 \%$} & \multicolumn{2}{|c|}{$1,45 \%$} & \multicolumn{2}{|c|}{$4,25 \%$} & \multicolumn{2}{|c|}{$5,25 \%$} \\
\hline & $\mu \mathrm{g} / \mathrm{ml}$ & $\% N$ cas. & $\mu \mathrm{g} / \mathrm{ml}$ & $\% \mathrm{~N}$ cas. & $\mu g / \mathrm{ml}$ & $\% N$ cas. & $\mu \mathrm{g} / \mathrm{ml}$ & $\% N$ cas. \\
\hline ... & 47 & 7,0 & 149 & 6,6 & 326 & 4,6 & 340 & 4,1 \\
\hline$\ldots$ & 32 & 4,8 & 102 & 4,6 & 218 & 3,1 & 238 & 2,9 \\
\hline $12 \ldots \ldots \ldots \ldots \ldots \ldots$ & 16 & 2,4 & 49 & 2,2 & 100 & 1,5 & 107 & 1,3 \\
\hline $21,5 \ldots \ldots \ldots \ldots \ldots \ldots$ & 8 & 1,2 & 28 & 1,2 & 40 & 0,6 & 41 & 0,5 \\
\hline $\begin{array}{l}\text { (Avant action de la pré- } \\
\text { sure ) de } 2 \text { à } 21,5 \% \text { TCA }\end{array}$ & 5 & 0,7 & 7 & 0,3 & 10 & 0,15 & 18 & 0,2 \\
\hline
\end{tabular}

Pour une concentration donnée de TCA, la quantité d'azote non protéique (qui est en relation avec la vitesse de la réaction), par $\mathrm{ml}$ de solution de caséine, varie beaucoup avec la concentration de cette dernière. Elle parait être proportionnelle à la concentration du substrat pour des solutions à 0,45 et $\mathrm{I}, 45 \mathrm{p}$. Ioo ; pour les solutions plus concentrées : 4,25 et 5,25 p. Ioo, elle est relativement moins grande. En conséquence la proportion de NPN p. Ioo N-caséine est moins élevée dans ces solutions concentrées. On peut supposer qu'il y a inhibition par excès de substrat ou que le NPN est en partie adsorbé sur le précipité.

\section{III. - Influence du mode de précipitation par l'acide trichloracétique sur la quantité d'azote non protéique}

I. - On peut vérifier la dernière explication ci-dessus en modifiant les conditions de la déprotéinisation par TCA de manière que la même quantité de protéine soit présente dans le mélange, quelle que soit la concentration de la caséine dans la solution emprésurée. Dans les expériences précédentes le volume des différentes 
solutions de caséine était le même (conditions A), donc la quantité de protéine dans le mélange avec TCA variait beaucoup. Dans les nouvelles expériences que nous avons faites, le volume de chaque solution de caséine, mélangé avec TCA, est inversement proportionnel à la concentration de la caséine (conditions B).

Le tableau 2 résume les résultats d'expériences comparatives, à I2 et à 2 p. roo de TCA. On voit que le mode de déprotéinisation a une grande influence lorsque la concentration finale de TCA est I2 p. Ioo. Les proportions d'azote non protéique trouvées dans les conditions habituelles (A) avec les solutions à 4,4, 2,2 et I,I p. roo de caséine sont: $I, 8,2,3$ et 3 , $\mathrm{xp}$. roo; alors que dans les conditions (B) (donnant

TABLEAU 2

Influence du mode de précipitation par l'acide trichloracétique

\begin{tabular}{|c|c|c|c|c|c|c|c|c|}
\hline & \multicolumn{4}{|c|}{$12 \%$ TCA } & \multicolumn{4}{|c|}{$2 \%$ TCA } \\
\hline & \multicolumn{2}{|c|}{$\underset{\text { filtrat }}{\mu \mathrm{g} N / \mathrm{ml}}$} & \multicolumn{2}{|c|}{$\begin{array}{l}\text { NPN \% } \\
\text { N-caséine }\end{array}$} & \multicolumn{2}{|c|}{$\begin{array}{l}\mu \mathrm{g} N / \mathrm{ml} \\
\text { filtrat }\end{array}$} & \multicolumn{2}{|c|}{$\begin{array}{l}\text { NPN \% } \\
\text { N-caséine }\end{array}$} \\
\hline & A & B & $\mathrm{A}$ & $\mathrm{B}$ & A & B & A & B \\
\hline $\begin{array}{l}\text { Augmentation de NPN après } 90 \mathrm{mn} \\
\text { d'action de la présure * sur les } \\
\text { solutions de caséine à : }\end{array}$ & & & & & & & & \\
\hline $4,4 \% \ldots \ldots \ldots \ldots \ldots \ldots \ldots$ & 23,7 & 18,6 & 1,8 & 1,3 & 54,1 & 54,0 & 4,0 & 3,8 \\
\hline $2,2 \% \ldots \ldots \ldots \ldots \ldots \ldots$ & 15,5 & 19,1 & 2,3 & 1,4 & 32,4 & 67,6 & 4,7 & 5,0 \\
\hline $1,1 \% \ldots \ldots \ldots \ldots \ldots \ldots$ & 10,3 & 19,1 & 3,1 & $1,4_{4}^{\prime}$ & 19,6 & 87,5 & 5,8 & 6,5 \\
\hline $\begin{array}{l}\text { NPN-O (avant l'action de la présure) } \\
\text { Solution } \grave{a}:\end{array}$ & & & & & & & & \\
\hline $4,4 \% \ldots \ldots \ldots \ldots \ldots$ & 3,8 & $3,{ }_{4}$ & 0,27 & 0,25 & 4,7 & 5,6 & 0,34 & 0,44 \\
\hline $2,2 \% \ldots \ldots \ldots \ldots$ & 2,3 & 3,1 & 0,36 & 0,23 & 4,4 & 5,6 & 0,70 & 0,44 \\
\hline $1,1 \% \ldots \ldots$ & 1,7 & 3,1 & 0,48 & 0,23 & 5,7 & 6,0 & 1,67 & 0,45 \\
\hline
\end{tabular}

A et $B$ : Voir le texte.

* : 0,1 UP, soit $1,35 \mu \mathrm{g} / \mathrm{ml}$.

le même volume de précipité) la même proportion est obtenue avec les trois solutions: I,3, I,4 et I,4 p. Ioo. Il est donc probable qu'intervient l'adsorption de substances solubles sur le précipité.

On ne constate pas de telles différences lorsque la concentration de TCA est 2 p. Ioo ; le précipité est d'ailleurs plus dispersé (sous forme de flocons), qu'à I2 p. Ioo de TCA.

2. - L'influence des conditions dans lesquelles la protéine, ayant subi l'action d'un enzyme, est séparée des produits solubles de la réaction a été mise en évidence par JACOBSEN (I947). Cet auteur a obtenu une plus forte proportion de substances solubles, en ajoutant de l'acétone à l'acide trichloracétique. Nous avons essayé cette addition. La figure 2 montre que la précipitation à $12 \mathrm{p}$. Ioo de TCA et Io $p$. Ioo d'acétone donne en effet une proportion d'azote non protéique plus élevée que la précipitation habituelle, avec TCA seul. Dans ce dernier cas, la valeur 
finale ne représente que de 79 à 86 p. Ioo de la valeur obtenue en présence d'acétone. La valeur du NPN-O est aussi légèrement plus faible sans acétone (0,I9 p. Ioo de $\mathrm{N}$-caséine) qu'en présence d'acétone (o,23 p. roo).

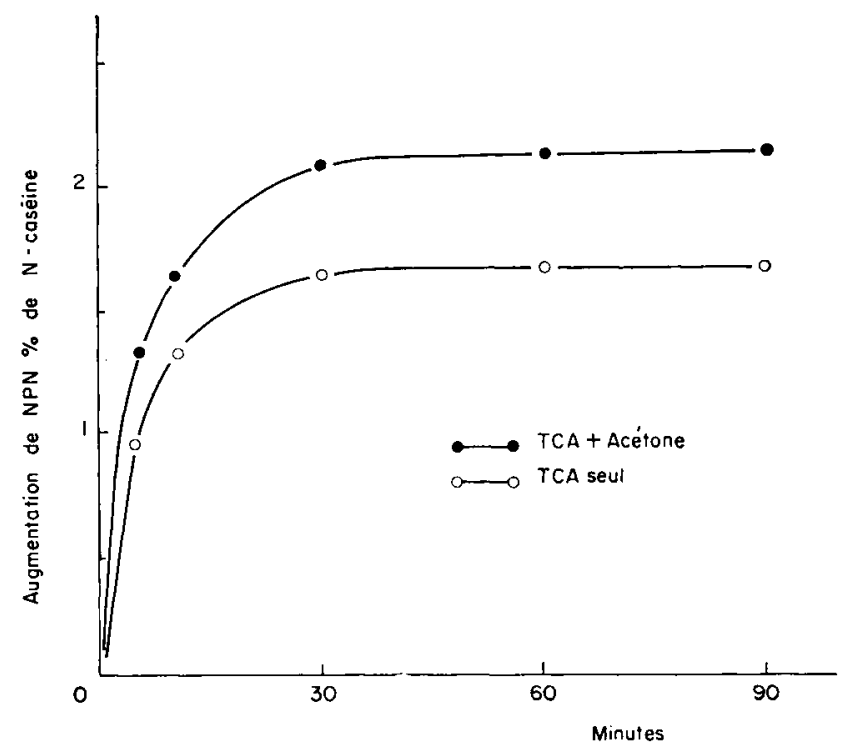

FIG. 2. - Courbes de VPN avec et sans acétone

$e: 0,65 \mu \mathrm{g} / \mathrm{ml}$ (présure cristallisée) ;

$s: 20 \mathrm{mg} / \mathrm{ml}$ (caséine entière);

pII 6,$8 ; 25^{\circ} \mathrm{C}$

TCA : $12 \%$

En ce qui concerne la précipitation avec 2 p. Ioo de TCA, on ne constate aucune différence, que le réactif contienne de l'acétone ou qu'il n'en contienne pas.

IV. - Formation de l'azote non protéique à partir de la caséine $\alpha$ et de la caséine $x$

\section{A. Caséine $\alpha$.}

La caséine $\alpha$ préparée par la méthode à l'urée est exempte des fractions $\beta$ et $\gamma$. Nous savons qu'elle n'est pas homogène; elle renferme la caséine $x$, insensible au calcium, et la caséine $\alpha$, sensible au calcium, et, peut-être, des constituants mineurs mal connus.

La présure réagit avec la caséine $\alpha$ de la même façon qu'avec la caséine entière (NrTSCHMANN et KELLER, I955). Toutefois, dans nos expériences, nous avons obtenu des proportions d'azote non protéique à $\mathrm{I} 2 \mathrm{p}$. IoO de TCA plus variables qu'avec la caséine entière, dans des conditions fixes de déprotéinisation. Après 60 minutes d'action de $0,03 \mathrm{UP} / \mathrm{ml}$, et en ajoutant I volume de TCA à $24 \mathrm{p}$. Ioo à I volume de solution de caséine à 3 . p. Ioo, ces proportions sont:

$I^{r e}$ préparation de caséine $\alpha, N P N=0,86$ et $I, I 2 p$. roo de $\mathrm{N}$-caséine. $2^{\mathrm{e}}$ préparation de caséine $\alpha, \mathrm{NPN}=\mathrm{I}, 67$ et $\mathrm{I}, 92 \mathrm{p}$. Ioo de $\mathrm{N}$-caséine 
Dans les mêmes conditions, la caséine entière donne de I,5 à I,9 p. Ioo de NPN. Les résultats ci-dessus permettent de penser que le traitement de la caséine entière, aboutissant à la séparation d'une fraction homogène à l'électrophorèse à $\mathrm{pH} 8,6$ et de mobilité $-6,5$, laisse une quantité variable, dans la caséine $\alpha$, du composant qui est le vrai substrat de l'enzyme.

\section{B. Caséine $x$}

Avec la caséine $x$, nous avons obtenu des courbes de NPN $f(t)$ à I2 p. Ioo de TCA de forme comparable à celles de la caséine entière et de la caséine $\alpha$; mais la proportion des produits solubles, après l'accomplissement de la réaction primaire, est environ 5 fois plus élevée qu'avec la caséine entière (tableau 3 ).

TABLEAU 3

Formation de NPN à partir de la caséine $\varkappa$

\begin{tabular}{|c|c|c|c|c|c|c|}
\hline \multirow{3}{*}{ Caséine $x$} & \multirow{3}{*}{$s^{*}$} & \multirow{3}{*}{$e^{*}$} & \multicolumn{4}{|c|}{ NPN \% de N caséine } \\
\hline & & & \multicolumn{2}{|c|}{ à $12 \% \mathrm{TCA} * *$} & \multicolumn{2}{|c|}{ à $2 \%$ TCA $* * *$} \\
\hline & & & Temps 0 & $\begin{array}{l}\text { Augmentation } \\
\text { après } 60 \mathrm{mn}\end{array}$ & Temps 0 & $\begin{array}{l}\text { Augmentation } \\
\text { après } 60 \mathrm{mn}\end{array}$ \\
\hline 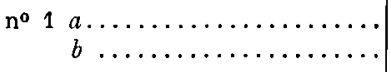 & $\begin{array}{l}1,56 \\
1,49\end{array}$ & $\begin{array}{l}0,05 \\
0,05\end{array}$ & $\begin{array}{l}1,15 \\
1,63\end{array}$ & $\begin{array}{l}8,95 \\
8,10\end{array}$ & & \\
\hline 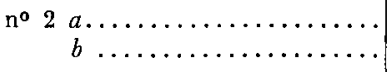 & $\begin{array}{l}1,27 \\
1,25\end{array}$ & $\begin{array}{l}0,03 \\
0,05\end{array}$ & 0,75 & 8,00 & $\begin{array}{l}3,2 \\
2,5\end{array}$ & $\begin{array}{l}15,0 \\
16,6\end{array}$ \\
\hline $\begin{array}{c}\text { Caséine entière (qui a servi à pré- } \\
\text { parer la caséine no } 2 \text { ) } \ldots . \ldots . .\end{array}$ & 1,33 & 0,03 & 0,21 & 1,55 & 0,3 & 3,65 \\
\hline
\end{tabular}

* $s$ : concentration de la caséine $: \mathrm{g} / 100 \mathrm{ml} . e$ : concentration de la présure cristallisée : UP/ml.

** 1 volume de TCA $24 \%$ ajouté à 1 volume de solution caséine $x$.

*** 1 volume de TCA $4 \%$ ajouté à 1 volume de solution de caséine $\varkappa$.

Le fait que la caséine $x$ subit une dégradation beaucoup plus importante que les caséines plus complexes dont elle est issue, est en faveur de la conception du substrat spécifique de l'enzyme, dans la réaction primaire.

Si l'on admet que les substances azotées non protéiques (à I2 p. roo de TCA) proviennent entièrement de la caséine $x$, les proportions indiquées dans le tableau 3 (caséine $\mathrm{n}^{\mathrm{o}}$ 2) permettent de calculer que la caséine entière doit contenir 19 p. Ioo de caséine $x(\mathrm{I}, 55 \times \mathrm{IOO} / 8)$.

Avec la caséine $x$, comme avec la caséine entière, la proportion d'azote non protéique formé dépend de la concentration du substrat. De même, à 2 p. Ioo de TCA ii y a environ deux fois plus d'azote non protéique qu'à I2 p. Ioo.

La valeur " NPN-O » est toujours plus élevée avec la caséine $x$ qu'avec la caséine entière ou la caséine $\alpha$. La solubilité apparente de la caséine $x$ dans TCA n'est donc pas négligeable. 
Il est à noter qu'avec la caséine $x$, la concentration de l'azote non protéique dans les filtrats à $\mathrm{I} 2 \mathrm{p}$. I00 de TCA (jusqu'à $0, \mathrm{I} 00 \mathrm{mg} / \mathrm{ml}$ ) est bien supérieure à celle que l'on a constatée avec la caséine entière (jusqu'à $0,050 \mathrm{mg} / \mathrm{ml}$ ) dans les mêmes conditions expérimentales. S'il s'agit, dans les deux cas, de la même substance, on peut en déduire que le filtrat trichloracétique obtenu avec la caséine entière n'est pas saturé en NPN et que le palier de la courbe caractéristique représente bien l'achèvement de la réaction primaire (figure $\mathrm{I}, 2,3$ ).

\section{V. - Phosphore et acide sialique solubles dans l'acide trichloracétique après l'action de la présure}

L'étude du passage en solution trichloracétique du phosphore et de l'acide sialique, détachés de la caséine après l'action de la présure, comparativement au passage en solution de l'azote, peut apporter des éclaircissements sur la nature des substances solubles.

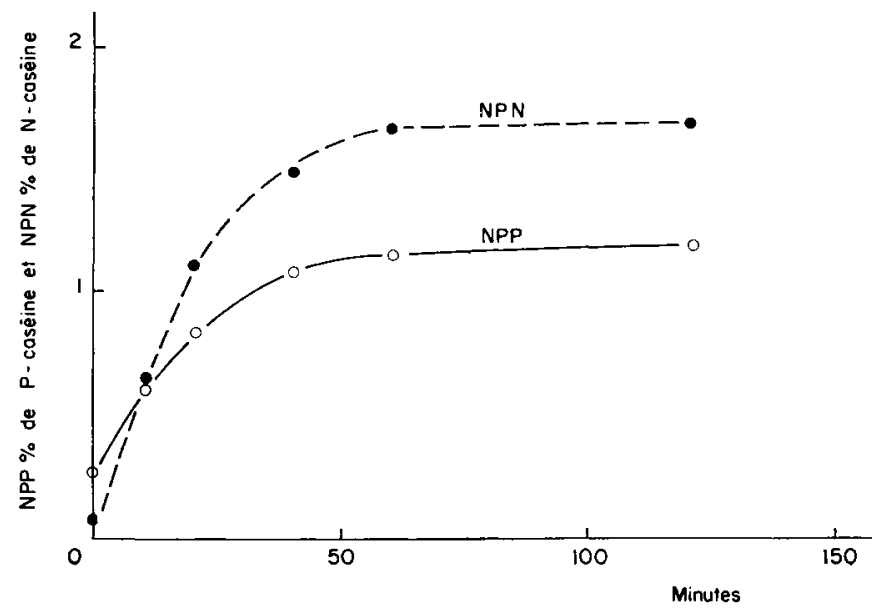

FIG. 3. - Phosphore non protéique (NPP) libéré par la présure
$s: 25 \mathrm{mg} / \mathrm{ml}$ (caséine $\alpha$ de vache);
$\mathrm{e}: 0,27 \mu \mathrm{g} / \mathrm{ml}$ (présure cristallisée);
pH 6,$8 ; 25^{\circ} \mathrm{C}$.

a) Dans 4 expériences, avec la caséine entière et la caséine $\alpha$ de vache et avec la caséine de chèvre, nous avons déterminé le phosphore et l'azote dans le filtrat trichloracétique obtenu en précipitant le mélange caséine + présure, après divers temps de réaction. La figure 3 montre les résultats d'une de ces expériences avec la caséine de vache. la courbe représentant la libération de phosphore non protéique soluble dans TCA à I 2 p. Ioo a la même forme que la courbe de l'azote non protéique ; mais elle ne lui est pas parallèle.

Avant l'addition de présure, la proportion de phosphore passant en solution est toujours plus élevée que celle de l'azote; apès l'action de la présure on constate le contraire. La rapport $\mathrm{P} / \mathrm{N}$ dans le filtrat trichloracétique va en diminuant régulièrement au cours de la réaction, en partant du temps zéro. Mais si l'on ne tient compte que du phosphore et de l'azote passés en solution après l'addition de la pré- 
sure, ce rapport reste sensiblement constant, comme le montre le tableau 4 et il est toujours nettement inférieur à ce qu'il est dans la caséine originelle.

On remarque que la petite fraction de la caséine, qui est solubilisée par I2 p. Ioo de TCA (sans action de la présure) est beaucoup plus riche en phosphore que le NPN proprement dit et que la caséine. Si cette fraction avait la même teneur en azote que la caséine ( $\mathrm{r}_{4}, 8 \mathrm{p}$. Ioo), elle contiendrait respectivement : $2,7-\mathrm{I}, 9$ et $3,6 \mathrm{p}$. Ioo de phosphore, dans le cas des 3 caséines du tableau 4.

TABLEAU 4

Phosphore non protéique (NPP) formé au cours de l'action de la présure*

\begin{tabular}{|c|c|c|c|c|c|c|c|c|}
\hline & \multirow{3}{*}{$\begin{array}{c}\mathrm{P} / \mathrm{N} \\
\text { caséine }\end{array}$} & \multicolumn{5}{|c|}{$\mathrm{P} / \mathrm{N}$ dans le filtrat trichloracétique } & \multicolumn{2}{|c|}{ NPP \% P-caséine } \\
\hline & & \multirow{2}{*}{$\begin{array}{c}\text { Temps } \\
0\end{array}$} & \multicolumn{4}{|c|}{ Après action de la présure $* *$} & \multirow{2}{*}{$\underset{0}{\text { Temps }}$} & \multirow{2}{*}{$\begin{array}{l}\text { Augmen- } \\
\text { tation } \\
\text { après } 60 \mathrm{mn}\end{array}$} \\
\hline & & & $15 \mathrm{mn}$ & $40 \mathrm{mn}$ & $60 \mathrm{mn}$ & $120 \mathrm{mn}$ & & \\
\hline \multicolumn{9}{|l|}{$\begin{array}{l}\text { 1) TCA } 12 \% \\
\text { Caséine de vache : }\end{array}$} \\
\hline $\begin{array}{l}\text { Caséine de vache : } \\
-\quad \text { entière .... }\end{array}$ & 0,056 & 0,17 & 0,030 & 0,032 & 0,035 & 0,032 & 0,25 & 0,87 \\
\hline$-\alpha \ldots \ldots \ldots \ldots \ldots \ldots$ & 0,062 & 0,12 & 0,040 & 0,042 & 0,041 & 0,040 & 0,29 & 1,02 \\
\hline $\begin{array}{l}\text { Caséine de chèvre } \ldots \ldots \ldots \ldots \\
\text { 2) TCA } 2 \% \\
\text { Caséine de vache : }\end{array}$ & 0,058 & 0,23 & 0,050 & 0,048 & 0,049 & - & 0,28 & 1,51 \\
\hline - entière............. & 0,056 & 0,05 & 0,036 & 0,038 & $0,03^{\prime}$ & 0,030 & 0,40 & 1,39 \\
\hline
\end{tabular}

* - $0,03 \mathrm{LP}$ soit $0,4 \mathrm{\mu g} / \mathrm{ml}$

** - Valeurs au temps 0 déduites.

A $2 \mathrm{p}$. Ioo de TCA, une expérience avec la caséine entière de vache montre que le rapport $P / N$, après action de la présure, est voisin de ce qu'il est à $I 2$ p. Ioo mais qu'au temps o il est beaucoup plus faible.

b) Nous avons suivi, en fonction du temps d'action de la présure, la quantité d'acide sialique, exprimée en acide $\mathrm{N}$-acétylneuraminique (NANA), se retrouvant dans le filtrat trichloracétique, avec la caséine $x$ comme substrat. Nous avons obtenu des résultats présentés dans le tableau 5, qui vont en sens contraire des précédents, en ce qui concerne les proportions.

TABLEAU 5

Acide sialique (NANA) non protéique formé au cours de l'action de la présure

\begin{tabular}{|c|c|c|c|c|c|c|c|c|}
\hline & \multirow{3}{*}{$\begin{array}{l}\text { NANA/N } \\
\text { caséine }\end{array}$} & \multicolumn{5}{|c|}{ NANA/N dans le filtrat trichloracétique } & \multicolumn{2}{|c|}{$\begin{array}{l}\text { NANA soluble } \% \\
\text { de NANA caséine }\end{array}$} \\
\hline & & \multirow{2}{*}{$\begin{array}{c}\text { Temps } \\
0\end{array}$} & \multicolumn{4}{|c|}{ Après action de la présure } & \multirow{2}{*}{$\begin{array}{c}\text { Temps } \\
0\end{array}$} & \multirow{2}{*}{$\begin{array}{l}\text { Augment. } \\
\text { apr. } 60 \mathrm{mn}\end{array}$} \\
\hline & & & $5 \mathrm{mn}$ & $15 \mathrm{mn}$ & $30 \mathrm{mn}$ & $60 \mathrm{mn}$ & & \\
\hline \multicolumn{9}{|l|}{ Caséine $\varkappa\left({ }^{*}\right)$} \\
\hline TCA $12 \% \ldots$ & 0,17 & 0,21 & 1,16 & 1,20 & 1,18 & 1,19 & 2,68 & 64,0 \\
\hline TCA $2 \%$ & 0,17 & 0,23 & 0,64 & 0,56 & 0,57 & 0,57 & 6,60 & 79,4 \\
\hline
\end{tabular}

(*) Solution à $0,4 \mathrm{~g} / 100 \mathrm{ml}$. 
Le rapport $\mathrm{NANA} / \mathrm{N}$ varie très peu, la valeur au temps zéro étant déduite, comme dans le cas du rapport $\mathrm{P} / \mathrm{N}$. Mais, dans le cas de l'acide sialique, la présure détache une ou des substances considérablement plus riches que la caséine originelle et que la fraction soluble au temps zéro.

Les courbes de formation de l'acide neuraminique ont la même allure que celles du NPN. Elles atteignent un palier horizontal avec I2 p. Ioo de TCA. Mais la proportion de NANA solubilisé est considérablement plus élevée que celle de l'azote.

\section{DISCUSSION}

L'interprétation des courbes de libération de l'azote non protéique soluble dans I2 p. roo d'acide trichloracétique, en fonction du temps d'action de la présure, a déjà été discutée (AlAIs et al., I953). La question se pose, en effet, de savoir si ces courbes traduisent bien la réaction primaire. La relation que nous avons constatée entre la libération de l'azote non protéique et l'évolution du temps de coagulation du lait constitue un argument en faveur de cette hypothèse.

Les résultats que nous avons obtenus, en faisant varier la concentration de la caséine, montrent que la vitesse de la libération des substances solubles n'est proportionnelle à la concentration du substrat que pour les faibles valeurs de cette dernière, comme le montre la figure 4 (d'après les données du tableau I).

Ce phénomène se produit aussi bien lorsque la concentration en acide trichloracétique est élevée : 12 et 22 p. Ioo (dans ce cas le filtrat trichloracétique ne contient que le caséino-glycopeptide), que lorsqu'elle est faible : 2 à 6 p. Ioo (dans ce cas d'autres substances solubles, résultant de la réaction de protéolyse générale, se trouvent présentes à côté du caséino-glycopeptide). Une inhibition de la réaction primaire par excès de substrat ne paraît pas devoir être retenue, pour expliquer les constatations précédentes. Les résultats présentés dans le tableau 2 montrent que lorsque la quantité de protéine dans le mélange à déprotéiniser ne varie pas, la quantité d'azote non protéique ne varie pas non plus, à partir de solutions de caséine à différentes concentrations.

Lorsqu'on étudie une réaction enzymatique de protéolyse, comme nous avons tenté de le faire pour la présure et la caséine, il faut tenir compte du fait que les méthodes usuelles de précipitation ne permettent pas une nette différenciation entre les trois types de substances: 1a protéine native, la protéine dégradée et les fragments libérés dont la solubilité dans les réactifs est plus ou moins grande.

Les expériences précédentes montrent que la proportion des substances solubles dépend (en dehors de la concentration de l'enzyme et du substrat dans le mélange réactionnel) :

I. - de la concentration de l'acide trichloracétique dans le mélange en cours de déprotéinisation.

2. - de la concentration de la caséine dans ce même mélange.

3. - des conditions expérimentales : manière de mélanger l'acide trichloracétique, présence d'acétone dans l'acide trichloracétique.

Il faut cependant remarquer que, lorsque l'on fait varier les conditions 2 et 3 , à I2 p. Ioo de TCA, la courbe de formation de l'azote non protéique en fonction du 
temps conserve la même forme ; seule change la valeur finale. Il est probable que l'adsorption de substances solubles sur le précipité est responsable de la diminution de cette valeur finale. Cette adsorption est d'autant plus grande que la quantité de caséine est plus élevée dans le mélange et que les conditions de l'expérience favorisent la formation d'un précipité plus compact.

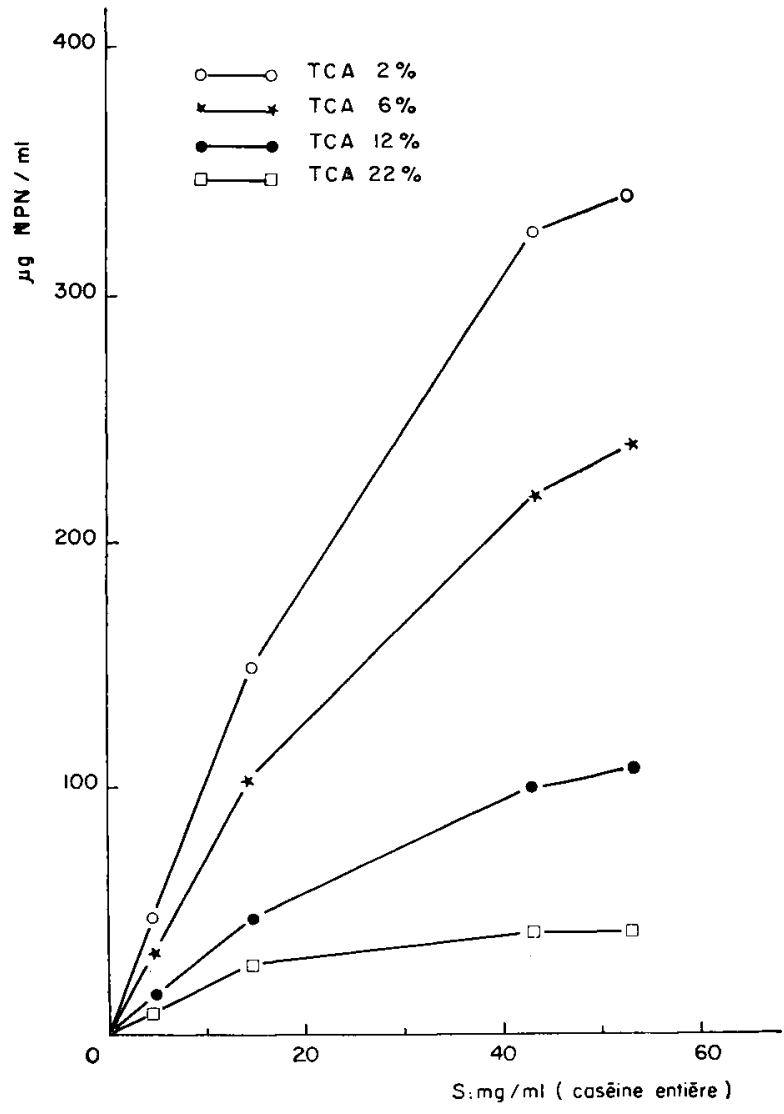

FIs. .4 - Quantité de NPN d différentes concentrations de casétne et d'acide trichloracétique $\mathrm{pH} 6,8 ; 25^{\circ} \mathrm{C}$. $e: 4 \mu \mathrm{g} / \mathrm{ml}$ (présure cristallisée).

Différents auteurs ont suivi la libération de l'azote non protéique à partir de la caséine $x$; leurs résultats, comparés aux nôtres, sont résumés dans le tableau 6 . L'augmentation de l'azote soluble, après l'action de la présure (au début du palier de la courbe NPN f $(t)$ ), est le plus souvent voisine de 8 p. roo. La valeur obtenue par GARNIER (I957) est beaucoup plus faible, mais sa " fraction $x$ " présente une solubilité élevée dans I2 p. Ioo de TCA (NPN-O) et une teneur en phosphore beaucoup plus haute que celle que l'on observe dans les préparations les plus pures de caséine $x$. 
Les résultats que nous avons obtenus avec la caséine $x$ sont intéressants pour deux raisons. D'une part, la valeur du NPN-O est faible; or la substance qui reste en solution lorsque l'on précipite la caséine $\varkappa$ par I 2 p. Ioo de TCA est très différente du caséino-glycopeptide libéré par la présure (communication à paraître) ; plus la proportion de cette substance (NPN-O) est élevée, plus l'interprétation des résultats analytiques concernant les substances solubles après l'action de la présure est difficile. D'autre part, nous avons étudié parallèlement, dans les mêmes conditions et à la même concentration, la libération de l'azote non protéique à partir de la caséine entière ; ce qui permet de calculer que la caséine entière de vache que nous avons utilisée doit contenir I9 p. Ioo de caséine $x$, valeur confirmée par la composition glucidique des caséines (Alais, I962). WaUGh et V. HiPPEi, (I956) ont calculé une proportion de I5 p. Ioo d'après les diagrammes d'électrophorèse.

TABLEAU 6

Libération de l'azote non protéique à partir de la caséine *

\begin{tabular}{|c|c|c|c|c|c|c|c|}
\hline Auteurs & $c(\mathbf{1})$ & $e(\mathbf{2})$ & $N P N-0\left({ }^{3}\right)$ & NPN (4) & $\mathrm{pH}$ & ${ }^{\circ} \mathrm{C}$ & $\mathrm{P} \%$ \\
\hline GARNIER (1957) & $0,1$. & 3,3 & 8,2 & 3,0 & - & 30 & 0,58 \\
\hline $\mathrm{W}_{\Lambda \mathrm{KE}}(1957) \ldots \ldots \ldots \ldots \ldots \ldots$ & 1,0 & 0,66 & 1,2 & 6,7 & 6,7 & 30 & 0,20 \\
\hline Tsugo et Yamauchi (1959) ... & 0, , $^{\prime}$ & 20,0 & 4,9 & 8,7 & 6,6 & 35 & 0,44 \\
\hline Befery et Nitscimmann $(1963) \ldots \ldots$ & 0,5 & $0,1 / 4$ & 2,5 & 8,0 & 7,0 & 25 & - \\
\hline Nos résultats .................... & 1,3 & $0,{ }^{\prime}$ & 0,75 & 8,0 & 6,7 & 25 & 0,22 \\
\hline
\end{tabular}

(1) $c$ : concentration du substrat, caséine $x$ p. 100 (p:v).

$\left(^{2}\right) e$ : concentration de l'enzyme, $\mu_{r}^{r}$ de présure cristallisée par $m l$ de solution de caséine $x$.

(3) Azote soluble dans 12 p. 100 de 'TCA avant l'action de la présure, en p. 100 de l'azote de la caséine.

(4) Azote soluble dans 1: p. 100 de TCA après l'action de la présure, en p. 100 de l'azote de la caséine, NPN-O déduit.

Les résultats concernant le phosphore et l'acide sialique montrent que la présure détache des fragments solubles très différents du substrat. Par rapport à la caséine originelle, le NPN est appauvri en phosphore et fortement enrichi en acide sialique. Le phosphore et l'acide sialique sont libérés à la même vitesse que l'azote nonprotéique ; dans le filtrat trichloracétique les. rapports $\mathrm{P} / \mathrm{N}$ et $\mathrm{NANA} / \mathrm{N}$ sont constants au cours de 1'action de la présure. Il est donc possible qu'une seule substance (le caséino-glycopeptide) soit libérée. Des résultats différents ont été publiés par $\mathrm{BEEBY}$ (I963) ; mais cet auteur ne déduit pas les valeurs au temps $O$.

La relation que nous avons constatée entre la libération de l'azote non-protéique et le temps de coagulation du lait d'une part, et l'analogie que présentent entre elles les courbes de libération de l'azote, du phosphore et de l'acide sialique non protéiques, avec une vitesse initiale élevée, sans période d'induction, dans des conditions de déprotéinisation où le caséino-glycopeptide apparaît seul, d'autre part, permettent de conclure que cette substance est le résultat direct de la réaction étudiée et que celleci correspond à la réaction primaire de la présure sur la caséine, qui est la cause de la coagulation. 


\section{SUMMARY}

\section{RELEASE OF NON-PROTEIN NITROGEN, PHOSPHORUS AND SIALIC ACID DURING RENNIN ACTION}

Non-protein substances are released from casein during the primary reaction; they are separated from the reaction mixture by trichloracetic acid at a final concentration of 2 or $12 \mathrm{p}$. 100 ; at the latter concentration " caseino-glycopeptide " is the only soluble product.

While studying the release of non-protein nitrogen soluble in 12 p. 100 trichloracetic acid from milk, a relationship was observed during the rennin action at $2^{\circ} \mathrm{C}$ between proteolysis and the clotting time of milk subjected to a rapid pre-heating. The clotting time decreases with an increase in non-protein nitrogen until no more is released, and at this point coagulation is almost instantaneous (fig. I).

The amount of non-protein nitrogen released by rennin from casein solutions is proportional to the amount substrate only when this is low (table I, fig. 4). The reaction does not appear to be inhibited by an excess of substrate. The amount of non-protein nitrogen in the trichloracetic filtrate depends on experimental conditions. The adsorption of soluble substance by the precipitate explains the anomalies observed (table 2, fig. 2).

It has been possible to calculate that there are I9 p. I00 xcasein in isoelectric whole casein (table 3 ) by comparing the results obtained using $x$-casein and the whole casein from which it was derived. The results obtained by different workers for the amount of non-protein nitrogen from $x$-casein is discussed (table 6).

The non-protein part soluble in $12 \mathrm{p}$. 100 trichloracetic acid released by rennin is low in phosphorus and rich in sialic acid. These components are released at the same rate as nitrogen (after the values at zero-times have been deducted). It is possible that a single substance is present.

It may be concluded from these results that the reaction resulting in the release of caseinoglycopeptide is definitively the primary reaction of rennin necessary for the ultimate coagulation.

\section{RÉFÉRENCES BIBLIOGRAPHIQUES}

Alais C., Mocouot G., Nitschmann Hs., Zahler P. I953. Das Lab und seine Wirkung auf das Casein der Milch. Helv. Chim. Acla, 36, 1955-1968.

Alais C. I955. Préparation de présure cristallisée à partir d'estomacs de veau. Ann. Technol. agric., 4, I 13 -I 20.

AlaIs C. 1956. Étude des substances azotées non protéiques séparées de la caséine sous l'action de la présure $X I V^{\circ}$ Congr. Intern. Laiterie, 2, 823-839.

Alais C. 1962. Étude de l'action enzymatique de la présure sur la caséine. Thèse. Paris.

Bamann E., Noworny E., Rohr L., I948. Zur colorimetrischen Bestimmung der Phosphorsäure. Chem. Ber., 81, 438-44I.

BEEBY R. I963. Studies on $x$-casein complex. I. The release of sialic acid-containing material by rennin. J. Dairy Res., $30,77-80$

BeEby R., Nitschmann Hs. I963. The action of rennin on casein. The disruption of $x$-casein complex. J. Dairy Res., 30, 7-I2.

BerRIDgE N. J., I942. The second phase of rennet coagulation. Nature, 149, I94-I95.

Garnier J.rg47. Fraction protéique dégradée spécifiquement par la présure dans la caséine. Intern. Symp. on Enzyme Chem., Tokyo, 524-526.

Hammarsten O. 1874. Über den chemischen Verlauf bei der Gerinnung des Caseins mit Lab. Maly's Jahresberichte $f$. Tierchem., 4, $135^{-1} 45$.

Hipp N. J., Groves M. L., Custer J. H., McMeekin T. L. I952. Separation of $\alpha$-, $\beta$ - and $x$-caseins. J. Dairy Sci., 35, 272-28r.

JACOBSEN C. F. 1947 . The activation of chymotrypsinogen. C. R. Lab. Carlsberg, sér. chim., 25, 327-437

Mc Kenzie H. A., Wake R. G., ig6r. An improved method for the isolation of $x$-casein. Biochim. Biophys. Acta., 4\%, 240-242. 
Nitschmann Hs., Keller W. I955. Das Lab und seine Wirkung auf das Casein der Milch. Helv. Chim. Acta, 38, 942-946.

Ribadeau Dumas B., Alais C. 196r. Préparation de l'acide N-acétylneuraminique cristallisé à partir de la caséine de vache. Bull. Soc. Chim. Biol., 43, 377-385.

Svennerholm L., I958. Quantitative estimation of sialic acids. Acta Chim. Scand., 12, 547-554.

Tsugo T., Yamauch $K$. 1960. Specific Liberation of non-protein nitrogen from $x$-casein by rennin and pepsin.Bull. Agric. Chem. Soc. Japon., 24, 96-100.

WAKE R. G. 1957. Studies on casein. V, The action of Rennin on casein. Austr. J. Sci., 20, I67.

WARREN L. r959. The thiobarbituric acid assay of sialic acid. J. Biol. Chem., 234, 1971-1975.

WAUGH D. F., v. HippeL P. H. 1956. $x$-casein and the stabilisation of casein micelles. J.Am.Chem. Soc., ${ }^{798}, 4576-4582$. 